\title{
On the Information Propagation Process in Multi-lane Vehicular Ad-hoc Networks
}

\author{
Zijie Zhang*, Guoqiang Mao*‡ and Brian D. O. Anderson ${ }^{\dagger \ddagger}$ \\ *School of Electrical and Information Engineering, University of Sydney, Australia \\ ${ }^{\dagger}$ Research School of Engineering, Australian National University, Australia \\ ${ }_{\ddagger}^{\ddagger}$ National ICT Australia (NICTA), Australia \\ Email: \{zijie.zhang, guoqiang.mao\}@sydney.edu.au, brian.anderson@anu.edu.au
}

\begin{abstract}
This paper studies the information propagation process in a 1D mobile ad-hoc network formed by vehicles traveling on a highway. We consider that vehicles can be divided into traffic streams; vehicles in the same traffic stream have the same speed distribution, while the speed distributions of vehicles in different traffic streams are different. Analytical formulas are derived for the fundamental properties of the information propagation process as well as the information propagation speed. Using the formulas, one can straightforwardly study the impact on the information propagation speed of various parameters such as radio range, vehicular traffic density, vehicular speed distribution and the time variation of vehicular speed.
\end{abstract}

\section{INTRODUCTION}

This paper studies the information propagation speed (IPS), which is the expected propagation speed for a piece of information to be broadcast along the road in a vehicular adhoc network (VANET). The IPS is an important performance metric for many VANET applications, especially for the safety messaging applications [1].

It has been shown [2], [3] that a VANET is usually partitioned into a number of clusters, where a cluster is a maximal set of vehicles in which every pair of vehicles is connected by at least one multi-hop path. Due to the mobility of vehicles, the clusters are splitting and merging over time. Therefore, information propagation in a VANET is typically based on a store-and-forward scheme. Considering the example illustrated in Fig. 1, a piece of information starts to propagate from the origin toward the positive direction of the axis at time $t_{0}$. The vehicles that have received this piece of information are referred to as the informed vehicles, where other vehicles are uninformed. The multi-hop forwarding of the message within a cluster, which begins at $t_{0}$ and ends at $t_{1}$, is called a forwarding process. In a forwarding process the information propagation speed is determined by the per-hop delay and the length of the cluster. The per-hop delay $\beta$ is the time required for a vehicle to receive and process a message before

This research is partially supported by ARC Discovery project DP110100538. This material is based on research partially sponsored by the Air Force Research Laboratory, under agreement number FA2386-10-1-4102. The U.S. Government is authorized to reproduce and distribute reprints for Governmental purposes notwithstanding any copyright notation thereon. The views and conclusions contained herein are those of the authors and should not be interpreted as necessarily representing the official policies or endorsements, either expressed or implied, of the Air Force Research Laboratory or the U.S. Government. it is available for further retransmission [4]. The value of $\beta$ reflecting the typical technology is $4 \mathrm{~ms}$ [4]. We show that the per-hop delay has a significant impact on the IPS.

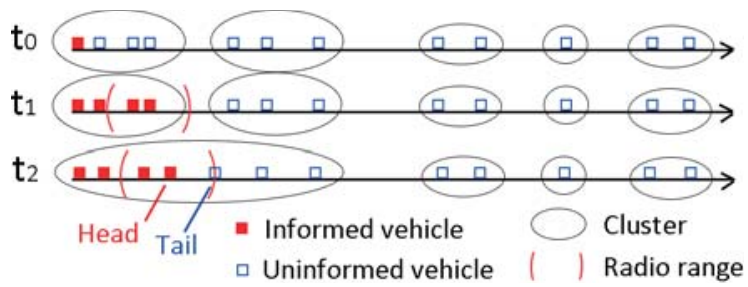

Fig. 1. Illustration of the topology of a VANET at different time instants. The positive direction of the axis is the direction of information propagation.

Define the head at time $t$ as the informed vehicle with the largest coordinate at time $t$. Define the tail at time $t$ as the uninformed vehicle with the smallest coordinate at time $t$. Two vehicles can directly communicate with each other if and only if their Euclidean distance is not larger than the radio range $r_{0}$, i.e. we adopt the unit disk model. As shown in Fig. 1, at time $t_{1}$ the tail is outside the radio range of the head. Then a catchup process begins, during which the informed vehicles hold the information until the head catches up the tail (at time $t_{2}$ ).

Recent research has shown that downstream traffic (a set of vehicles traveling in the opposite direction of information propagation) can be exploited to improve the IPS [2], [5], [6]. Further, real world measurements show that vehicles traveling in different lanes (e.g. bus lane or heavy truck lane) have different speed distributions [7]. In view of these observations, we consider multiple traffic streams, where a traffic stream is a set of vehicles following the same speed distribution. A traffic stream does not have to consist of vehicles traveling in the same lane. Traffic streams also represent different types of vehicles (e.g. sports cars or heavy trucks). Interesting results are obtained by allowing vehicles in different traffic streams to have different speed distributions.

Main contributions of this paper are: firstly, an analytical model for the information propagation process considering multiple traffic streams is provided. It is shown that a small difference in the average vehicular speeds between traffic streams can result in a significant increase of the IPS. Secondly, time variation of vehicular speed is considered in the analysis, which results in interesting conclusions, e.g. the IPSs are similar for information propagating in both positive and 
negative directions of a road, which is different from previous studies (e.g. [5], [6]) considering time-invariant vehicular speed only. Based on the analysis of the catch-up process and forwarding process, analytical results for the IPS are derived, which are validated using simulations. This paper provides useful guidelines on the design of a multi-lane VANET.

The rest of this paper is organized as follows: Section II reviews related work. Section III introduces the mobility model and network model. The analysis on the catch-up process is given in Section IV. The forwarding process is studied in Section V, followed by the results of the IPS. Section VI validates the analysis using simulations. Section VII concludes this paper and discusses future work.

\section{RELATED WORK}

In [2], Agarwal et al. studied the IPS in a 1D VANET where vehicles are Poissonly distributed and move at the same speed, which is time-invariant, but either in the positive (upstream) or negative (downstream) direction of the axis. The upstream and downstream traffic have the same density. This work was later extended in [5] to allow the upstream and the downstream traffic to have different densities. The authors derived upper and lower bounds for the IPS, which provided a hint on the impact of vehicle density on the IPS. Recent research of Baccelli et al. [6] provided analytical results on the IPS under the same setting as that in [5]. Note that the analyses in [2], [5], [6] were all based on the simplifying assumption that vehicles in each traffic stream travel at the same speed, which is time-invariant. Evidently, in such model, catch-up can only occur via the vehicles travelling in the opposite direction.

In [3], Wu et al. considered a 1D VANET where vehicles are Poissonly distributed and the vehicle speeds are uniformly distributed within a designated range. Considering one traffic stream and time-invariant vehicular speed, they provided analytical results on the IPS when the vehicle density is either very low or very high. They also provided a numerical method to calculate the IPS in a VANET with two traffic streams. In an earlier work [8], we showed that the time-variation of vehicular speed has a significant impact on the IPS. However, only one traffic stream was considered in [8], i.e. all vehicles travel in the same direction and follow the same speed distribution. This paper considers multiple traffic streams and evaluating the impact of vehicular densities and speed distributions on information propagation in multi-lane VANETs.

\section{System Model}

Suppose that there are a total of $N$ traffic streams, wherein vehicles are free to change lanes and overtake other vehicles. We adopt the commonly-used traffic model in traffic theory [9], viz. the number of vehicles in the $n^{\text {th }}$ traffic stream passing an observation point during any time interval follows a homogeneous Poisson process with intensity $\lambda_{n} \mathrm{veh} / \mathrm{s}$.

A synchronized random walk mobility model is considered. Specifically, time is divided into time slots with equal length $\tau$. Then, the $i^{t h}$ time slot is $t \in((i-1) \tau, i \tau]$. Each vehicle in the $n^{\text {th }}(n \in[1, N])$ traffic stream chooses its speed randomly at the beginning of each time slot, independent of the speeds of other vehicles and its own speed in other time slots, according to a probability density function (pdf) $f_{v n}(v)$. In this paper, we consider the Gaussian speed distribution, i.e. $f_{v n}(v) \sim \mathcal{N}\left(\mu_{n}, \sigma_{n}^{2}\right)$, where $\mu_{n}$ (resp. $\left.\sigma_{n}^{2}\right)$ is the mean speed (resp. variance) in the $n^{\text {th }}$ traffic stream. The Gaussian speed distribution is commonly used for modeling the VANETs on a freeway [3], [9], [10].

Under the aforementioned setting, it can be shown that at any time instant the spatial distribution of the vehicles in the $n^{\text {th }}$ traffic stream follows a homogeneous Poisson process with intensity $\rho_{n}=\lambda_{n} \int_{-\infty}^{\infty} \frac{f_{v n}(v)}{v} d v$ [8]. Then according to the superposition property of the Poisson process, at any time instant the spatial distribution of all the vehicles on the road follows a homogeneous Poisson process with intensity $\rho=\sum_{n=1}^{N} \rho_{n}$.

\section{CATCh-UP PROCESS}

Without loss of generality, it is assumed that the catch-up process starts at time 0 . The displacement $x(x \in(-\infty, \infty))$ of a vehicle at time $t$ is defined to be the distance between the position of the vehicle at time 0 and its position at time $t$.

This section is organized as follows: in Lemma 1, we study the movement of a single vehicle. The result on the distance between two vehicles at a given time is summarized in Lemma 2. After considering the overtaking of vehicles in Section IV-C, we obtain the delay for a catch-up process in Section IV-D.

\section{A. Modeling the movement of a single vehicle}

Denote by $p_{n}(x, t)$ the probability that the displacement of a vehicle in the $n^{\text {th }}$ traffic stream is $x$ at time $t$. We have:

Lemma 1: Under the system model introduced in Section III, when $t=i \tau$,

$$
p_{n}(x, t)=p_{n}(x, i \tau)=\frac{1}{\tilde{\sigma}_{i} \sqrt{2 \pi}} \exp \left(\frac{-\left(x-\mu_{n} i \tau\right)^{2}}{2 \tilde{\sigma}_{i}^{2}}\right)
$$

where $\tilde{\sigma}_{i}^{2}=i\left(\sigma_{n} \tau\right)^{2}$.

Proof: According to Gaussian speed distribution model, the pdf of the speed of a vehicle in the $n^{\text {th }}$ traffic stream is:

$$
f_{v n}(v)=\frac{1}{\sigma_{n} \sqrt{2 \pi}} \exp \left(\frac{-\left(v-\mu_{n}\right)^{2}}{2 \sigma_{n}^{2}}\right)
$$

Because the speed does not change during a time slot, it is straightforward that $p_{n}(x, \tau)$ is also a Gaussian function:

$$
p_{n}(x, \tau)=\frac{1}{\sigma_{n} \tau \sqrt{2 \pi}} \exp \left(\frac{-\left(x-\mu_{n} \tau\right)^{2}}{2\left(\sigma_{n} \tau\right)^{2}}\right)
$$

Due to the independence of the vehicular speeds in different time slots (hence the displacements), we have for $t=i \tau$ :

$$
p_{n}(x, t)=p_{n}(x, i \tau)=\overbrace{\left(p_{n} * p_{n} * \ldots * p_{n}\right)(x, \tau)}^{\text {i-fold convolution }}
$$

Then one can obtain Eq. 1 using the property that the convolution of two Gaussian functions is a Gaussian function. 


\section{B. The distance between a pair of vehicles}

As shown in Fig. 2, denote by $H_{m}$ (resp. $P_{m}$ ) the $m^{\text {th }}$ vehicle to the left of the head $H_{0}$ (resp. to the right of the tail $\left.P_{0}\right)$ at time 0 . If $H_{m}$ happens to be in the $n^{\text {th }}$ traffic stream, which happens with a probability $\frac{\rho_{n}}{\rho}$, then an additional label $n$ (e.g. $H_{m}^{n}$ ) is used to indicate that the vehicle $H_{m}$ is in the $n^{\text {th }}$ traffic stream. In this subsection, we study the distance between $H_{m}^{n}$ and $P_{m^{\prime}}^{n^{\prime}}$, where $n, n^{\prime} \in[1, N]$ and $m, m^{\prime}$ are non-negative integers.

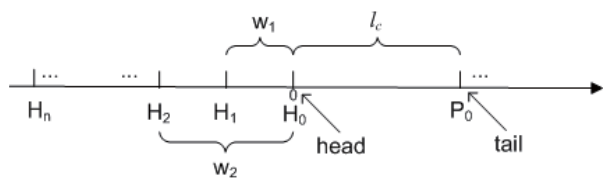

Fig. 2. Illustration of a VANET at the beginning of a catch-up process.

The catch-up process between $H_{m}^{n}$ and $P_{m^{\prime}}^{n^{\prime}}$ finishes as soon as the distance between them reduces to radio range $r_{0}$ for the first time. Note that the reduction of distance between $H_{m}^{n}$ and $P_{m^{\prime}}^{n^{\prime}}$ can reach a given value $z$ at several time instants with non-zero probabilities. We are interested in the probability that the reduction of the distance between $H_{m}^{n}$ and $P_{m^{\prime}}^{n^{\prime}}$ reaches $z$ for the first time, i.e. the first passage time phenomenon, which is essential for the analysis of the catch-up process.

Lemma 2: Denote by $G_{m m^{\prime}}^{n n^{\prime}}(z, i)$ the probability that the reduction of the distance between $H_{m}^{n}$ and $P_{m^{\prime}}^{n^{\prime}}$, with regards to their distance at time 0 , reaches $z(z \neq 0)$ for the first time in the $i^{t h}(i \geq 1)$ time slot. Then: (a) When $\mu_{n}=\mu_{n^{\prime}}$ :

$G_{m m^{\prime}}^{n n^{\prime}}(z, i)=\frac{z}{i \sqrt{2 \pi i \tau^{2}\left(\sigma_{n}^{2}+\sigma_{n^{\prime}}^{2}\right)}} \exp \left(\frac{-z^{2}}{2 i \tau^{2}\left(\sigma_{n}^{2}+\sigma_{n^{\prime}}^{2}\right)}\right)$

(b) When $\mu_{n} \neq \mu_{n^{\prime}}$ and $i=1$ :

$$
G_{m m^{\prime}}^{n n^{\prime}}(z, i) \leq \frac{1}{2}\left(1-\operatorname{erf}\left(\frac{z-\mu_{i}}{\sqrt{2 \sigma_{i}^{2}}}\right)\right)
$$

(c) When $\mu_{n} \neq \mu_{n^{\prime}}$ and $i \geq 2$ :

$$
G_{m m^{\prime}}^{n n^{\prime}}(z, i) \leq \frac{1}{4}\left(1+\operatorname{erf}\left(\frac{z-\mu_{i-1}}{\sqrt{2 \sigma_{i-1}^{2}}}\right)\right)\left(1-\operatorname{erf}\left(\frac{z-\mu_{i}}{\sqrt{2 \sigma_{i}^{2}}}\right)\right)
$$

where $\mu_{i}=\mu_{n} i \tau-\mu_{n^{\prime}} i \tau, \sigma_{i}^{2}=\left(\sigma_{n}^{2}+\sigma_{n^{\prime}}^{2}\right) \tau^{2} i$.

Proof: Denote by $g_{m m^{\prime}}^{n n^{\prime}}(z, t)$ the probability that the reduction of the distance between $H_{m}^{n}$ and $P_{m^{\prime}}^{n^{\prime}}$ is $z$ at time $t$. When $t=i \tau$,

$$
\begin{aligned}
g_{m m^{\prime}}^{n n^{\prime}}(z, t) & =\int_{-\infty}^{\infty} p_{n}(x, t) p_{n^{\prime}}(x-z, t) d x \\
& =\int_{-\infty}^{\infty} p_{n}(x, t) p_{n^{\prime}}(z-x, t) d x \\
& =\left(p_{n} * p_{n^{\prime}}\right)(x, t) \\
& =\frac{1}{\sqrt{2 \pi \sigma_{i}^{2}}} \exp \left(\frac{-\left(z-\mu_{t}\right)^{2}}{2 \sigma_{t}^{2}}\right)
\end{aligned}
$$

where $\mu_{t}=\left(\mu_{n}-\mu_{n^{\prime}}\right) t, \sigma_{t}^{2}=\left(\sigma_{n}^{2}+\sigma_{n^{\prime}}^{2}\right) \tau t$ and Eq. 11 is obtained from Eq. 10 using Lemma 1. Note that Eq. 9 is obtained from Eq. 8 by letting the mean speed of $P_{m^{\prime}}^{n^{\prime}}$ be 0 instead of $\mu_{n^{\prime}}$, and consequently the mean speed of $H_{m}^{n}$ becomes $\mu_{n}-\mu_{n^{\prime}}$. Therefore, $p_{n^{\prime}}(x, t)$ becomes a zero mean Gaussian function with respect to $x$ hence $p_{n^{\prime}}(x-z, t)=$ $p_{n^{\prime}}(z-x, t)$.

When two traffic streams have the same mean speed, i.e. $\mu_{n}=\mu_{n^{\prime}}$, we have a special case that $\mu_{t}=0$ in Eq. 11. For simplicity, the probability that the reduction of the distance between $H_{m}^{n}$ and $P_{m^{\prime}}^{n^{\prime}}$ reaches $z$ in the $i^{t h}$ time slot, i.e. $\int_{(i-1) \tau}^{i \tau} g_{m m^{\prime}}^{n n^{\prime}}(z, t) d t$ is approximated by $\tau g_{m m^{\prime}}^{n n^{\prime}}(z, i \tau)$. That is we approximately consider that $g_{m m^{\prime}}^{n n^{\prime}}(z, t)=g_{m m^{\prime}}^{n n^{\prime}}(z, i \tau)$ for $t \in((i-1) \tau, i \tau]$. Applying a standard procedure [8] to determine the first passage probability $G_{m m^{\prime}}^{n n^{\prime}}(z, i)$, we have:

$\tau g_{m m^{\prime}}^{n n^{\prime}}\left(z^{\prime}, i^{\prime} \tau\right)=\tau \sum_{i=1}^{i^{\prime}} G_{m m^{\prime}}^{n n^{\prime}}(z, i) g_{m m^{\prime}}^{n n^{\prime}}\left(z^{\prime}-z,\left(i^{\prime}-i\right) \tau\right)$

Solving the equation by the Z-transform as introduced in our previous work [8], one can obtain that for $\mu_{n}=\mu_{n^{\prime}}$ :

$$
G_{m m^{\prime}}^{n n^{\prime}}(z, i)=\frac{z}{i \sqrt{2 \pi i \tau^{2}\left(\sigma_{n}^{2}+\sigma_{n^{\prime}}^{2}\right)}} \exp \left(\frac{-z^{2}}{2 i \tau^{2}\left(\sigma_{n}^{2}+\sigma_{n^{\prime}}^{2}\right)}\right)
$$

When $\mu_{n} \neq \mu_{n^{\prime}}$, we use a method different from the complicated first passage analysis to calculate $G_{m m^{\prime}}^{n n^{\prime}}(z, i)$ from $g_{m m^{\prime}}^{n n^{\prime}}(z, t)$. Define $\mathcal{A}$ to be the event that the reduction of distance between $H_{m}^{n}$ and $P_{m^{\prime}}^{n^{\prime}}$ is smaller than $z$ at time $(i-1) \tau$. Define $\mathcal{B}$ to be the event that the reduction of distance between $H_{m}^{n}$ and $P_{m^{\prime}}^{n^{\prime}}$ is larger than $z$ at time $i \tau$. Then for $\mu_{n} \neq \mu_{n^{\prime}}$ and $i \geq 2$ :

$$
\begin{aligned}
& G_{m m^{\prime}}^{n n^{\prime}}(z, i) \leq \operatorname{Pr}(\mathcal{A}) \operatorname{Pr}(\mathcal{B} \mid \mathcal{A}) \leq \operatorname{Pr}(\mathcal{A}) \operatorname{Pr}(\mathcal{B}) \\
& =\int_{0}^{z} g_{m m^{\prime}}^{n n^{\prime}}\left(z_{0},(i-1) \tau\right) d z_{0} \int_{z}^{\infty} g_{m m^{\prime}}^{n n^{\prime}}\left(z_{0}, i \tau\right) d z_{0} \\
& =\frac{1}{2}\left(1+\operatorname{erf}\left(\frac{z-\mu_{i-1}}{\sqrt{2 \sigma_{i-1}^{2}}}\right)\right) \frac{1}{2}\left(1-\operatorname{erf}\left(\frac{z-\mu_{i}}{\sqrt{2 \sigma_{i}^{2}}}\right)\right)
\end{aligned}
$$

where $\mu_{i}=\mu_{n} i \tau-\mu_{n^{\prime}} i \tau, \sigma_{i}^{2}=\left(\sigma_{n}^{2}+\sigma_{n^{\prime}}^{2}\right) \tau^{2} i$. The first inequality is due to the fact that we do not consider the first passage phenomenon. Hence $\operatorname{Pr}(\mathcal{A}) \operatorname{Pr}(\mathcal{B} \mid \mathcal{A})$ can be larger than the probability that the reduction of distance between $H_{m}^{n}$ and $P_{m^{\prime}}^{n^{\prime}}$ reaches $z$ for the first time in the $i^{\text {th }}$ time slot. This bound is fairly tight in the case $\mu_{n} \neq \mu_{n^{\prime}}$ that we considered here. Because when $\mu_{n} \neq \mu_{n^{\prime}}, g_{m m^{\prime}}^{n n^{\prime}}(z, t)$ becomes a Gaussian function with a non-zero mean according to Eq. 11. It follows that the mean reduction of distance between $H_{m}^{n}$ and $P_{m^{\prime}}^{n^{\prime}}$ becomes a decreasing (when $\mu_{n}<\mu_{n^{\prime}}$ ) or increasing (when $\mu_{n}>\mu_{n^{\prime}}$ ) function of $t$. Therefore the first passage phenomenon is less notable when $\mu_{n} \neq \mu_{n^{\prime}}$. Secondly, the bound $\operatorname{Pr}(\mathcal{B} \mid \mathcal{A}) \leq \operatorname{Pr}(\mathcal{B})$ in Eq. 14 is due to the observation that $\mathcal{A}$ and $\mathcal{B}$ are negatively correlated, i.e. given that the reduction of distance is less than $z$ at time $(i-1) \tau$, the reduction of distance is less likely to be larger than $z$ at time $i \tau$. Further, this correlation appears to have limited impact on the final result under the settings introduced in Section VI.

For $\mu_{n} \neq \mu_{n^{\prime}}$ and $i=1, \operatorname{Pr}(\mathcal{A})=1$. Therefore:

$$
G_{m m^{\prime}}^{n n^{\prime}}(z, i) \leq \operatorname{Pr}(\mathcal{B})=\frac{1}{2}\left(1-\operatorname{erf}\left(\frac{z-\mu_{i}}{\sqrt{2 \sigma_{i}^{2}}}\right)\right)
$$




\section{The catch-up process between $H_{m}$ and $P_{m^{\prime}}$}

A catch-up process, where the distance between the head $H_{0}$ and tail $P_{0}$ is $l_{c}$ at time 0 , is referred to as a catch-up process with gap $l_{c}$. Note that the head at time $t$ is not necessarily the head vehicle at time $0\left(H_{0}\right)$ because $H_{0}$ may be overtaken by another informed vehicle during time $(0, t]$. We first consider the catch-up process between a pair of vehicles $H_{m}-P_{m^{\prime}}$.

Denote by $q_{m m^{\prime}}\left(i \mid l_{c}\right)$ the probability that $H_{m}$ catches up $P_{m^{\prime}}$ for the first time in the $i^{t h}$ time slot, in a catch-up process with gap $l_{c}$. Recall that with probability $\frac{\rho_{n}}{\rho}$ (resp. $\frac{\rho_{n^{\prime}}}{\rho}$ ) that $H_{m}$ (resp. $P_{m^{\prime}}$ ) belongs to the $n^{\text {th }}$ (resp. $n^{\prime t h}$ ) traffic stream. It can be shown that

$$
q_{m m^{\prime}}\left(i \mid l_{c}\right)=\sum_{n, n^{\prime} \in[1, N]} G_{m m^{\prime}}^{n n^{\prime}}(z, i) \frac{\rho_{n} \rho_{n^{\prime}}}{\rho^{2}}
$$

where $z=l_{c}-r_{0}+w_{m}+w_{m^{\prime}}$ and $w_{m}$ (resp. $w_{m^{\prime}}$ ) is the expected distance between $H_{m}$ and $H_{0}$ (resp. $P_{m^{\prime}}$ and $P_{0}$ ) at time 0 as shown in Fig. 2. Due to the Poisson distribution of vehicles, the inter-vehicle distance follows an exponential distribution with mean $1 / \rho$. It follows that $w_{m}=m / \rho, w_{m^{\prime}}=$ $m^{\prime} / \rho$. Therefore $z=l_{c}-r_{0}+m / \rho+m^{\prime} / \rho$.

\section{Delay of a catch-up process}

Define the catch-up delay $t_{c}$ to be the time taken from the beginning of a catch-up process till the time when the head and tail move into the radio range of each other for the first time, i.e. $t_{2}-t_{1}$ in Fig. 1 . Denote by $H\left(i \mid l_{c}\right)$ the probability that none of the $H_{m}-P_{m^{\prime}}$ pairs catches up in the $i^{t h}$ time slot, in a catch-up process with gap $l_{c}$. Due to the independence between the movements of vehicles, we have:

$$
H\left(i \mid l_{c}\right)=\prod_{m, m^{\prime} \in[0, \infty)}\left(1-q_{m m^{\prime}}\left(i \mid l_{c}\right)\right)
$$

where $q_{m m^{\prime}}\left(i \mid l_{c}\right)$ is given by Eq. 18 .

Denote by $h\left(i \mid l_{c}\right)$ the probability that at least one pair of $H_{m}-P_{m^{\prime}}$ catches up in the $i^{t h}$ time slot and none of them has caught up before the $i^{\text {th }}$ time slot, in a catch-up process with gap $l_{c}$. Assume that the catch-up event in the $i^{\text {th }}$ time slot is independent of that in the $j^{\text {th }}$ time slot for $0<j<i$, which is an accurate approximation when the duration of a time slot is large, e.g. $\tau=1 \mathrm{~s}$ or $5 \mathrm{~s}$ as shown in Section VI. Then:

$$
h\left(i_{c} \mid l_{c}\right)=\left(1-H\left(i_{c} \mid l_{c}\right)\right) \prod_{i=1}^{i_{c}-1} H\left(i \mid l_{c}\right)
$$

Finally the expected delay for a catch-up process with gap $l_{c}$ is $E\left[t_{c} \mid l_{c}\right]=\sum_{i=1}^{\infty} i \tau h\left(i \mid l_{c}\right)$

\section{FORWARDING PROCESS AND IPS}

Define forwarding delay as the time required for a packet to be forwarded from the leftmost vehicle to the rightmost vehicle in a cluster. Assume that a cluster does not become disconnected during the forwarding process since the per-hop delay is relatively small (e.g. 4ms [4]). Then, the expected forwarding delay in a cluster with length $x_{0}$ is $E\left[t_{f} \mid x_{0}\right]=$ $\beta E\left[k \mid x_{0}\right]$, where $E\left[k \mid x_{0}\right]$ (given in [8]) is the expected number of hops between two vehicles separated by $x_{0}$.
The entire information propagation process can be considered as a renewal reward process where each cycle consists of a catch-up process followed by a forwarding process and the reward is the information propagation distance during each cycle. Therefore, the expected IPS $\left(E\left[v_{i p}\right]\right)$ is [3]:

$$
\begin{aligned}
& E\left[v_{i p}\right] \approx \frac{\text { expected length of one cycle }}{\text { expected time duration of one cycle }}+\mu_{\max } \\
& =\frac{\int_{r_{0}}^{\infty} l_{c} f_{l_{c}}\left(l_{c}\right) d l_{c}+\int_{0}^{\infty} x_{0} f_{x_{0}}\left(x_{0}\right) d x_{0}}{\int_{r_{0}}^{\infty} E\left[t_{c} \mid l_{c}\right] f_{l_{c}}\left(l_{c}\right) d l_{c}+\beta+\int_{0}^{\infty} E\left[t_{f} \mid x_{0}\right] f_{x_{0}}\left(x_{0}\right) d x_{0}} \\
& \quad+\mu_{\max }
\end{aligned}
$$

where $\mu_{\max }=\max _{n \in N}\left\{\mu_{n}\right\}$ is the maximum average speed among traffic streams and $f_{l_{c}}\left(l_{c}\right)=\rho e^{-\rho\left(l_{c}-r_{0}\right)}$ is the pdf of distance between two adjacent but disconnected vehicles [8].

\section{Simulation RESUlts}

Simulations are conducted in a VANET simulator written in $\mathrm{C}++$. Each point shown in the figures is the average value from 2000 simulations. The confidence interval is too small to be distinguishable and hence is ignored in the following plots. The radio range is $r_{0}=250 \mathrm{~m}$ [3]. The typical values of the mean and standard deviation of vehicular speed are $25 \mathrm{~m} / \mathrm{s}$ and $7.5 \mathrm{~m} / \mathrm{s}$ [10]. These mobility parameters, i.e. $\mu_{n}, \sigma_{n}$ and $\tau$, are taken from practical measurements, where the usual record time intervals for a vehicle speed monitor are $\tau=1 s, 5 s$ [11]. The traffic density is varied so that the density $\rho$ varies from 0 to $0.06 \mathrm{veh} / \mathrm{m}$. For completeness of the plot, $\rho=0$ is included which means there is only one vehicle in each traffic stream.

Fig. 3 and Fig. 4 show the expected IPS in a VANET. Firstly it can be seen that analytical results have a good match with simulation results. Secondly, when the vehicular density is low, the IPS is determined by vehicular speeds (whose mean value is $25 \mathrm{~m} / \mathrm{s}$ ) because there is little packet forwarding. Thirdly, when the vehicular density increases, small clusters are formed and the IPS increases. As the vehicular density further increases, clusters become larger and the forwarding process starts to dominate. Therefore, the IPS increases until it reaches the maximum value $r_{0} / \beta$, where $\beta$ is the per-hop delay. When the vehicular density is moderate, the IPS is determined by the catch-up delay, which is further determined by the mobility of vehicles, as shown in our previous work [8].

Moreover, vehicular speed distribution also has a significant impact on the catch-up process hence the IPS. Fig. 3 shows the IPS in a VANET with two traffic streams with equal vehicular density but different vehicular speed distributions. It can be seen that our analytical result has a better match with the simulation results than that in [6], which does not consider the catch-up process studied in Lemma 2 of this paper. An interesting observation is that the IPS increases when the average speed of the vehicles in one of the traffic streams is reduced from $25 \mathrm{~m} / \mathrm{s}$ to $0 \mathrm{~m} / \mathrm{s}$. Further, an even faster IPS is observed when the average speed of the vehicles in one of the traffic streams is $-25 \mathrm{~m} / \mathrm{s}$, i.e. two traffic streams head in opposite directions. The reason behind this interesting observation is that a larger relative speed between vehicles 
results in faster catch-up processes hence a faster IPS. This can also be seen from the analytical results of $G_{m m^{\prime}}^{n n^{\prime}}(z, i)$, e.g. applying the fact that the error function is an increasing function to Eq. 6. This observation tells us that making use of the vehicles in the negative traffic stream can increase the IPS. Moreover, some (stationary) roadside units without (expensive) wired connections can also significantly increase the IPS in a VANET.

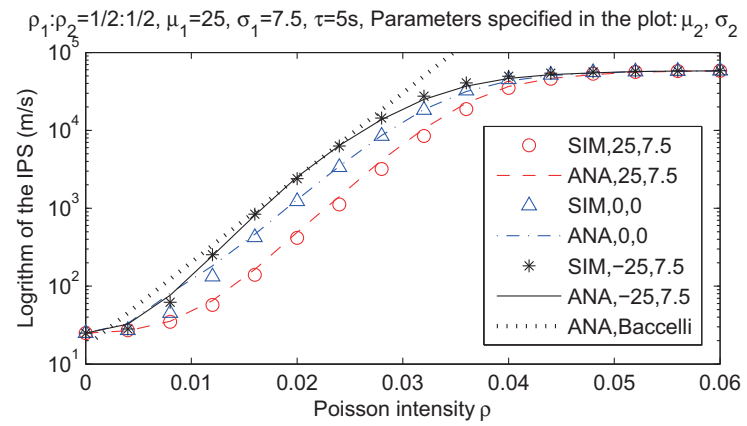

Fig. 3. The expected IPS in a VANET with different vehicular speeds in two traffic streams $(N=2)$. The plot Baccelli shows the IPS derived in [6] for a VANET with vehicular speed 25 and -25 in two streams respectively.

Fig. 4 shows the expected IPS in a VANET with different vehicular densities in two traffic streams. Firstly, it can be seen that an uneven distribution of vehicular densities between traffic streams (e.g. $\rho_{1}=\rho / 10, \rho_{2}=9 \rho / 10$ ) results in a slower IPS, compared with the IPS in a VANET with evenly distributed traffic densities (e.g. $\rho_{1}=\rho_{2}=\rho / 2$ ). This is because an uneven distribution of vehicular densities between traffic streams results in a smaller number of catchups between vehicles in different traffic streams (as manifested by the factor $\frac{\rho_{n} \rho_{n^{\prime}}}{\rho^{2}}$ in Eq. 18), hence less improvement on IPS is provided by the large relative vehicular speed, compared with a VANET with evenly distributed vehicular densities between traffic streams. This situation can be observed on freeways connecting the business district and residential district. Densities of the traffic streams in opposite directions can vary greatly depending on time-of-day when people going to work or coming back home. On the other hand, it can be seen that a small amount (e.g. $\rho / 10)$ of vehicular traffic in an opposite direction can still significantly increase the IPS. Therefore, it is meaningful to exploit the traffic streams with different mean speeds (e.g. heavy trucks or buses).

Moreover, it can be seen in Fig. 4 that the last four curves are very close to each other, which suggests that given a configuration of the vehicular densities and speed distributions in a VANET, the IPSs are similar for the information propagating in both positive and negative directions. This conclusion is different from previous ones that were based on the timeinvariant speed model [5], [6]. Our results suggest that a similar IPS is achievable in both directions of a two-way communication despite the uneven vehicular density between traffic streams.

\section{CONCLUSION AND FUTURE WORK}

In this paper, analytical results are provided for the information propagation process in a mobile ad-hoc network

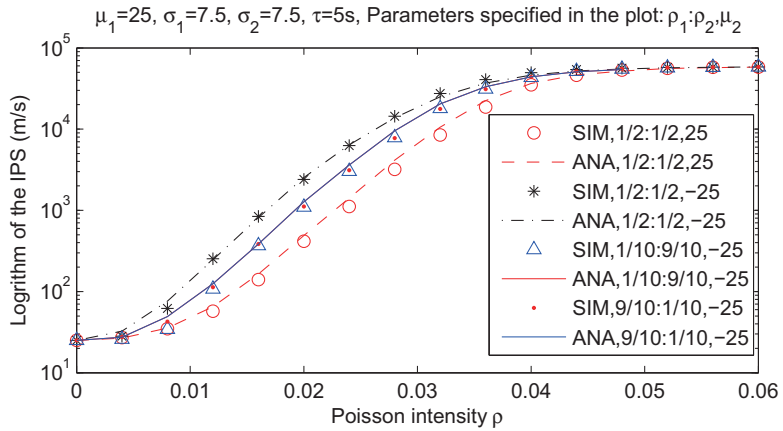

Fig. 4. The expected IPS in a VANET with different vehicular densities in two traffic streams. The first four curves are kept from Fig. 3 for comparison.

formed by vehicles moving on a freeway. It is considered that vehicles can be divided into multiple traffic streams. Vehicles in the same traffic stream have the same speed distribution while the speed distributions of vehicles in different traffic streams are different. We found that the IPS can be boosted significantly by exploiting the existence of even a small number of vehicles traveling with a different mean speed, such as vehicles traveling in the opposite direction or heavy trucks moving in the same direction but with a slower speed. On the other hand, the IPSs are similar for the information propagating toward both positive and negative directions in the case of uneven traffic densities among various traffic streams. By using parameters extracted from real world measurements for $\lambda, \mu, \sigma$ and $\tau$, our results can provide a quick estimate of the IPS with a good accuracy. The analysis can be further extended to 2D topologies in the future. Further, we are going to consider the impact of channel randomness on the IPS.

\section{REFERENCES}

[1] D. Camara, C. Bonnet, and F. Filali, "Propagation of public safety warning messages: a delay tolerant network approach," in Proceedings IEEE WCNC, 2010.

[2] A. Agarwal, D. Starobinski, and T. D. Little, "Analytical model for message propagation in delay tolerant vehicular ad hoc networks," in IEEE Vehicular Technology Conference, 2008, pp. 3067-3071.

[3] H. Wu, R. M. Fujimoto, G. F. Riley, and M. Hunter, "Spatial propagation of information in vehicular networks," IEEE Transactions on Vehicular Technology, vol. 58, no. 1, pp. 420-431, 2009.

[4] H. Wu, J. Lee, M. Hunter, R. Fujimoto, R. L. Guensler, and J. Ko, "Efficiency of simulated vehicle-to-vehicle message propagation in Atlanta, Georgia, I-75 corridor," Transportation Research Record: Journal of the Transportation Research Board, vol. 1910, pp. 82-89, 2005.

[5] A. Agarwal and T. D. Little, "Impact of asymmetric traffic densities on delay tolerant vehicular ad hoc networks," in IEEE Vehicular Networking Conference (VNC), 2009, pp. $1-8$.

[6] E. Baccelli, P. Jacquet, B. Mans, and G. Rodolakis, "Information propagation speed in bidirectional vehicular delay tolerant networks," in Proceedings IEEE INFOCOM, 2011, pp. 436-440.

[7] G. Yan, N. Mitton, and X. Li, "Reliable routing in vehicular ad hoc networks," in The 7th International Workshop on Wireless Ad hoc and Sensor Networking, Genoa, Italie, 2010.

[8] Z. Zhang, G. Mao, and B. D. Anderson, "On the information propagation speed in mobile vehicular ad hoc networks," in Proceedings IEEE GLOBECOM, 2010, pp. 1-5.

[9] W. Leutzbach, Introduction to the Theory of Traffic Flow. SpringerVerlag, 1988.

[10] M. Rudack, M. Meincke, and M. Lott, "On the dynamics of ad hoc networks for inter vehicle communications (IVC)," in Proceedings ICWN, 2002.

[11] "Carchip fleet pro," 2010. [Online]. Available: http://www.carchip.com 